\title{
Attitude control of microsatellite using PWM method: verification using PIL
}

\author{
Ho-Nien Shou \\ Department of Aviation \& Communication Electronics, No.198, Jieshou W. Rd., Gangshan District \\ Kaohsiung City, 82047, Taiwan (R.O.C.) \\ E-mail:honien.shou@gmail.com
}

\begin{abstract}
The missions of micro-satellites range from early military usages to weather forecast, resources exploration, communication, and scientific experiment. The advantages of micro-satellites are simple mechanical structures, great reliability, low prices, and precise researchers and equipment. Therefore, due to the limits of weight and power, this kind of micro-satellite with low design cost and high precision requires reducing unnecessary attitude sensors and controllers to ensure the precision of attitude control. Besides, it adapts the least hardware constituting attitude system, which is the future trend in the satellite engineering. In this article, an estimating technology about measuring angle speed with a gyro less is mentioned. This technology is based on the period change which the earth magnetic field gets along the track. Only by using a three axis magnetometer, it can produce the data from the micro-satellite measuring the earth magnetic field. Besides, the measures of three axis angle speed and attitude angle can be gotten through Kalman filtering. The purpose of this article is mainly to explore the problem on attitude detumbling control of micro-satellites departing away a carrier to enter a track. It is realized by a thruster to proceed the confinement of the satellite moving, the attitude stable control and processor-in-the-loop (PIL).
\end{abstract}

Keywords: three axis magnetometer, earth magnetic field, Kalman filtering, processor-in-the-loop.

\section{Introduction}

Before the satellite departs away the carrier into the mission track, it will tumble with a high speed rotating attitude. Or before the initial rotating speed exceeds the designed tolerant value, the function of the attitude control system lies to confine tumble and lower the angle speed for the satellite to achieve the pointed location. If the attitude control system uses a thruster to correct the attitude, the advantage is that it is beyond the limits of outer circumstances and easy to operate. In addition, it is suitable for any controlling methods, fast to correct time, and precise to point. 1) The uncertainties in the interior satellite are there is an error in the setting distance of the spray head, the inclined side of the spray head is not accurate, and the usage of gas fuel makes the quality center deflect. 2) The exterior disturbances of the satellite are the height and position to the environmental factors: gravity gradient, air resistance and magnetic forces. 3) Using the spray head easily produces bang-bang control. It is impossible as a linear switch to have high fuel efficiency, which can use the pulse-width pulsefrequency modulation to solve the thruster problem at the same time[1][2].

As to a micro-satellite with lower precision to three axis point, lighter carrier, low power consumption and limited research charge, there is undoubtedly a good inducement to merely use the magnetometer to complete the three axis attitude and the speed of the attitude angle. Therefore, it is practically important to develop this engineering. In the past, the magnetometer is often used as an assistance to meet with other parts in the satellite attitude confirming system. It is less to use a single magnetometer to complete attitude confirmation. The main reason is that the attitude precision is limited by using this method.

(C) The 2020 International Conference on Artificial Life and Robotics (ICAROB2020), Jan. 13-16, B-Con Plaza, Beppu, Oita, Japan 
It cannot get rid of the error limitation from incorrect terrestrial magnetism. This common method is to use the star pattern to get the track parameter and then pass through International Geomagnetic Reference Field (IGRF) to find our local geomagnetic field[3], which compares with the magnetometer measuring value to get the attitude angle by the attitude algorithm. However, by doing so, it only can get two axis attitude angles, but it cannot provide the message of the angle efficiency. To get satellite upper angle efficiency, it is necessary to use a gyroscope. However, the function of the gyroscope will be worsened with time, and its price is high and the equipment is complicated. Therefore, it may be a good choice to adapt attitude angle sensor instead of a gyroscope. In the article, the formula of micro-satellite and exercise is calculated by quaternion and angle speed. In it, an unscented Kalman filtering is adopted. The purpose of the article focuses on (1) Designing the thruster position, (2) Taking the satellite attitude angle speed and four-element position as an order of thruster, (3) Considering when there is an error in the distance of the thruster position, the uncertainty in the interior satellite is the quality center change by using gas fuels, (4) Exterior disturbances are: satellite height and position to magnetic force, gravity gradient, and air resistance, (5) Using the thruster with the pulse-width pulse-frequency modulation to solve the thruster problem. (6) Describing the above questions by the leaning differential inequality [4] [5], which means to reduce the exterior disturbances and limit the system parameter uncertainty of the satellite attitude controller.

The contents of the article are as follows: section II problem description, the Thrust force derived of satellite in section III, section IV Pulse-width Pulse-frequency Modulation Rules, section V Kalman Filtering and Different Calculating Methods, section VI Fulfillment of Software-in-the-Loop (SIL) and processor-in-the-Loop (PIL), and future prospects and conclusions in the last section.

\section{Problem Description}

Considering satellite attitude exercise formula

$$
\dot{\boldsymbol{q}}=\frac{1}{2}\left[\begin{array}{cc}
-\boldsymbol{\omega}_{o}^{b \times} & \boldsymbol{\omega}_{o}^{b} \\
-\boldsymbol{\omega}_{o}^{b T} & 0
\end{array}\right]\left[\begin{array}{c}
\mathbf{q} \\
q_{4}
\end{array}\right]
$$

Satellite non-linear dynamics formula

$$
(J+\Delta J) \boldsymbol{\omega}_{i}^{b}=-\boldsymbol{\omega}_{i}^{b \times}(J+\Delta J) \boldsymbol{\omega}_{i}^{b}+\boldsymbol{\tau}_{w}+\boldsymbol{\tau}_{u}
$$

$$
\text { (i) } \boldsymbol{q}=\left[\begin{array}{ll}
\mathbf{q}^{T} & q_{4}
\end{array}\right]^{T}=\left[\begin{array}{llll}
q_{1} & q_{2} & q_{3} & q_{4}
\end{array}\right]^{T}=\left[\begin{array}{ll}
\vec{e} \sin \frac{\phi}{2} & \cos \frac{\phi}{2}
\end{array}\right]^{T}
$$

The above is a quaternion, which is to rotate an Euler angle by order to a new attitude.

Suppose the specific condition is $\vec{e}=\left[\begin{array}{lll}e_{x} & e_{y} & e_{z}\end{array}\right]^{T}$ and the condition rotating angle is $\phi$.

(ii) $\omega_{o}^{b}$ is the angular velocity of the body frame with respect to the orbit frame. Among it,

$$
\boldsymbol{\omega}_{o}^{b}=\left[\begin{array}{lll}
\omega_{x} & \omega_{y} & \omega_{z}
\end{array}\right]^{T}, \quad \boldsymbol{\omega}_{o}^{b \times}=\left[\begin{array}{ccc}
0 & -\omega_{z} & \omega_{y} \\
\omega_{z} & 0 & -\omega_{x} \\
-\omega_{y} & \omega_{x} & 0
\end{array}\right] \text { is a }
$$

corresponding $\omega_{o}^{b}$ opposing skew matrix.

(iii) $\boldsymbol{\omega}_{i}^{b}$ is the angular velocity of the body frame with respect to the inertial frame.

(iv) $J+\Delta J$ is inertial matrix,

$$
J+\Delta J=\left[\begin{array}{lll}
J_{x x} & J_{x y} & J_{x z} \\
J_{x y} & J_{y y} & J_{y z} \\
J_{x z} & J_{y z} & J_{z z}
\end{array}\right]+\left[\begin{array}{ccc}
\Delta J_{x x} & \Delta J_{x y} & \Delta J_{x z} \\
\Delta J_{x y} & \Delta J_{y y} & \Delta J_{y z} \\
\Delta J_{x z} & \Delta J_{y z} & \Delta J_{z z}
\end{array}\right]
$$

In it, $J_{i i}$ is $i$ axle rotates inertial, and $\Delta J_{i j}$ is $i$ axle rotates inertial along $j$ axle. When the fuels are used, they will the quality center in the satellite system, which are uncertain items in the satellite system parameter.

(v) $\tau_{w}$ represents that the satellite will be disturbed by the force, including gravity gradient force, air resistance force, and magnetic disturbed force.

(vi) $\tau_{u}$ represents satellite attitude controlled force. In the paper, the control theory is conducted to get the relationship $\boldsymbol{\tau}_{u}$ thruster matrix and output $\boldsymbol{u}$, and then is combined pulse-width modulation and the jet to produce thrust.

\section{Production of Satellite Thrust}

The satellite exercise frame is as figure 1. Four thrusters are put $x-y$ plane, including $\lambda$ angle, the jet moves toward $-z$ direction, and lines to be a square distance $d$. Fuel is put the jet $x-y$ plane $l$ distance, and $+z$ direction is toward satellite center, in figure $2 . \Delta \lambda$ 、 $\Delta d$ and $\Delta l$ respectively represent the jet including angle, jet relative distance, and the uncertainty of the fuel gravity position. The following is production $\boldsymbol{\tau}_{u}$ from the thruster: 


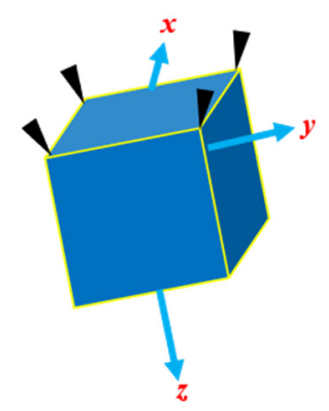

Figure 1 Satellite frame

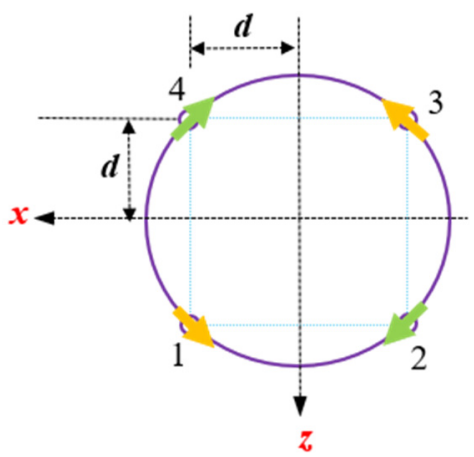

Figure 2 Satellite Jet Geometry Frame

$\boldsymbol{\tau}_{\boldsymbol{u}}=\left[\begin{array}{c}\tau_{x} \\ \tau_{y} \\ \tau_{z}\end{array}\right]=\left[\begin{array}{llll}\vec{r}_{1} \times \vec{F}_{1} & \vec{r}_{2} \times \vec{F}_{2} & \vec{r}_{3} \times \vec{F}_{3} & \vec{r}_{4} \times \vec{F}_{4}\end{array}\right] \boldsymbol{u}$

$$
=(\boldsymbol{B}+\Delta \boldsymbol{B}) \boldsymbol{u}
$$

$\begin{array}{llll}1 & 2 & 3 & 4\end{array}$

$\vec{r}=\left[\begin{array}{cccc}-(d+\Delta d) & -(d+\Delta d) & (d+\Delta d) & (d+\Delta d) \\ -(d+\Delta d) & (d+\Delta d) & (d+\Delta d) & -(d+\Delta d) \\ -(l+\Delta l) & -(l+\Delta l) & -(l+\Delta l) & -(l+\Delta l)\end{array}\right] \begin{gathered}x \\ y \\ z\end{gathered}$

$\left[\begin{array}{llll}-\rho \sin (\lambda+\Delta \lambda) & -\rho \sin (\lambda+\Delta \lambda) & \rho \sin (\lambda+\Delta \lambda) & \rho \sin (\lambda+\Delta \lambda) \\ \hline & \end{array}\right.$

$\vec{F}=\left[\begin{array}{cccc}\rho \sin (\lambda+\Delta \lambda) & -\rho \sin (\lambda+\Delta \lambda) & -\rho \sin (\lambda+\Delta \lambda) & \rho \sin (\lambda+\Delta \lambda) \\ -\cos (\lambda+\Delta \lambda) & -\cos (\lambda+\Delta \lambda) & -\cos (\lambda+\Delta \lambda) & -\cos (\lambda+\Delta \lambda)\end{array}\right] y$

$\left[\begin{array}{llll}-\cos (\lambda+\Delta \lambda) & -\cos (\lambda+\Delta \lambda) & -\cos (\lambda+\Delta \lambda) & -\cos (\lambda+\Delta \lambda)\end{array}\right] z$

$\alpha=\frac{1}{\sqrt{2}}, \beta_{1}=d+\alpha \lambda l, \beta_{2}=d-\alpha \lambda l, \beta_{3}=\frac{\lambda d}{\alpha}$,

$\Delta \beta_{1}=\Delta d-\lambda \Delta \lambda(d+\Delta d)+\alpha(\lambda \Delta l+l \Delta \lambda+\Delta \lambda \Delta l)$

$\Delta \beta_{2}=\Delta d-\lambda \Delta \lambda(d+\Delta d)-\alpha(\lambda \Delta l+l \Delta \lambda+\Delta \lambda \Delta l)$

$\Delta \beta_{3}=\frac{1}{\alpha}(\lambda \Delta d+d \Delta d+\Delta \lambda \Delta d)$

When the thruster transfers matrix $\boldsymbol{B}$, the features are as follows[6].

1) $\operatorname{rank}(\boldsymbol{B}+\Delta \boldsymbol{B})=\min \{m, n\}$

$$
\text { 2) } \begin{aligned}
& (\boldsymbol{B}+\Delta \boldsymbol{B})(\boldsymbol{B}+\Delta \boldsymbol{B})^{T} \\
= & {\left[\begin{array}{ccc}
4\left(\beta_{1}+\Delta \beta_{1}\right) & 0 & 0 \\
0 & 4\left(\beta_{2}+\Delta \beta_{2}\right) & 0 \\
0 & 0 & 4\left(\beta_{3}+\Delta \beta_{3}\right)
\end{array}\right] }
\end{aligned}
$$

\section{Pulse-width Modulation Rules}

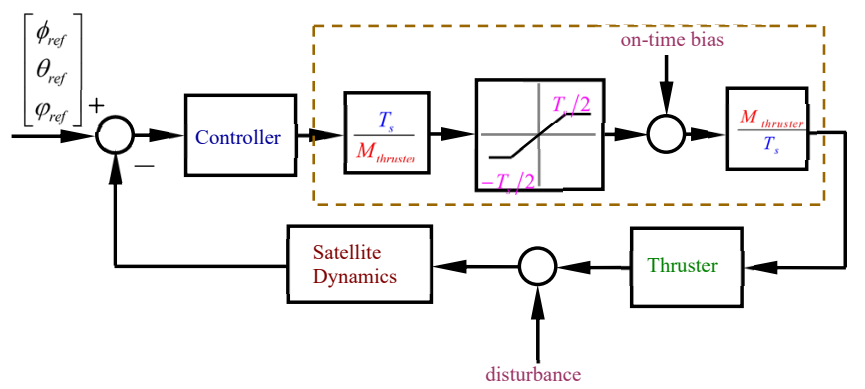

Figure 3 Micro-satellite Attitude Control Pulse-Width Modulation Thruster Block Diagram

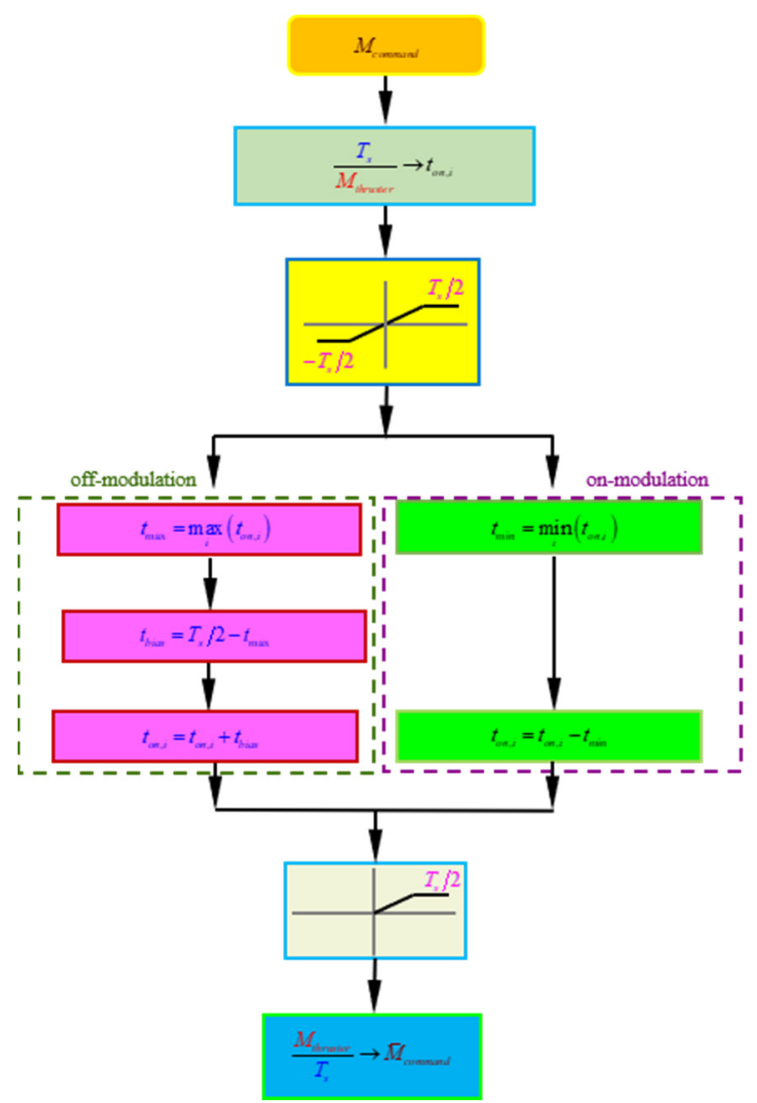

Figure 4 Pulse-width on/off modulation Control Process Chart

(C) The 2020 International Conference on Artificial Life and Robotics (ICAROB2020), Jan. 13-16, B-Con Plaza, Beppu, Oita, Japan 


\section{Running Heads}

Because the software loop cannot be completely tested in attitude measuring elements and the thruster, the Processor-in-the-Loop in the second stage is needed [6]. In figure 5, the structure of the Processor-in-the-Loop.

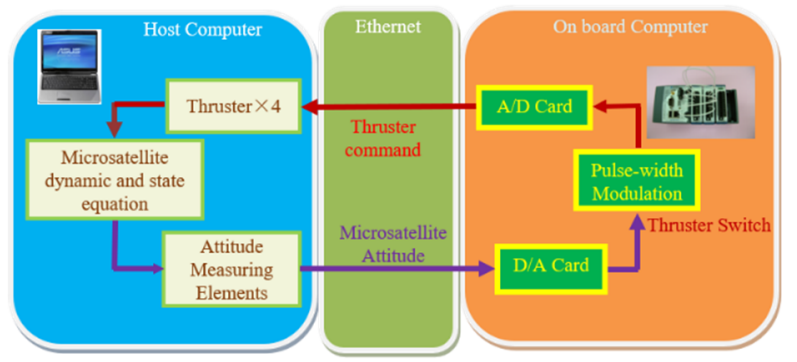

Figure 5 Processor-in-the-Loop system block diagram

TABLE I. PARAMETERS OF MICROSATELLITE

\begin{tabular}{|c|c|c|c|}
\hline Item & Uncertainty Bound & Value & Unit \\
\hline$\lambda$ & $\Delta \lambda / \lambda= \pm 0.1$ & 5 & $\mathrm{deg}$ \\
\hline$l$ & $\Delta l / l= \pm 0.1$ & 0.24 & $m$ \\
\hline$d$ & $\Delta d / d= \pm 0.1$ & 0.25 & $m$ \\
\hline$J_{x x}$ & $\Delta J_{x x} / J_{x x}= \pm 0.1$ & 5.5384 & $\mathrm{~kg}-\mathrm{m}^{2}$ \\
\hline$J_{y y}$ & $\Delta J_{y y} / J_{y y}= \pm 0.1$ & 5.6001 & $\mathrm{~kg}-\mathrm{m}^{2}$ \\
\hline$J_{z z}$ & $\Delta J_{z z} / J_{z z}= \pm 0.1$ & 4.2382 & $\mathrm{~kg}-\mathrm{m}^{2}$ \\
\hline
\end{tabular}

TABLE II. INITIAL CONDITIONS OF MICROSATELLITE

\begin{tabular}{|c|c|c|c|}
\hline Item & Description & Value & Unit \\
\hline$[\varphi, \theta, \psi]$ & Initial Euler Angle & {$\left[\begin{array}{llll}-120 & 30 & 60\end{array}\right]$} & deg \\
\hline $\begin{array}{r}{\left[q_{1}, q_{2},\right.} \\
\left.q_{3}, q_{4}\right]\end{array}$ & Initial Quaternion & $\begin{array}{c}{[0.0474} \\
0.5303- \\
0.6597 \\
0.5303]\end{array}$ & \\
\hline$\left[\omega_{x}, \omega_{y}, \omega_{z}\right]$ & $\begin{array}{c}\text { Initial Angular } \\
\text { Rate }\end{array}$ & {$\left[\begin{array}{lll}3.5 & -3.5 & 5.23\end{array}\right]$} & $\mathrm{rad} / \mathrm{s}$ \\
\hline$d t$ & Step Time & 1.0 & sec \\
\hline$T_{\text {sim }}$ & Simulation Time & 1200 & $\sec$ \\
\hline
\end{tabular}

The content of the experiment, will use the satellite parameters of Table I and Table II the initial conditions to simulate the satellite attitude response.

\section{Acknowledgements}

This work was supported by the National Science Council of Republic of China under contract NSC 1072221-E-344-002 and the construction technological advice, assistance, and financial support from National Space Center research project NSPO-S-099135.

\section{References}

1. J. Yuan, D. Yang, and H. Wei, " Flexible Satellite Attitude Maneuver Control Using Pulse-width Pulsefrequency Modulated Input Shaper," International Symposium on Systems and Control in Aerospace and Astronautics, pp.1407-1412, 2006.

2. B. N. Agrawal, R. S. Mcclelland and G. Song, "Attitude Control of Flexible Spacecraft Using Pulse-Width PulseFrequency Modulated Thrusters," Space Technolog, vol. 17, No. 1, pp.15-34, 1997.

3. H. Weidong, Z. Yulin, "Rate Damping Control for Small Satellite Using Thruster," Acta Astronautica, vol.55, pp.913, 2004.

4. [4] Ho-Nien, Shou and J, C, Juang, "An LMI-Based Nonlinear Attitude Control Approach,” IEEE Transactions on Control Systems Technology, vol.11, no. 1, pp.73-83, 2003.

5. Ho-Nien, Shou and J, C, Juang, Y. W. Jan and C. T. Lin, "Quaternion Feedback Attitude Control Design: A Nonlinear Approach," Asian Journal of Control, vol. 5, no. 3, pp. 406-411, 2003.

6. Ho-Nien Shou, "A Two-Phase Optimization Algorithm in Controller Synthesis," Intelligent Engineering Systems through Artificial Neural Networks, Issue 5, pp.1 12, 2011. 